University of Wollongong

Research Online

Faculty of Engineering and Information

Faculty of Engineering and Information

Sciences - Papers: Part A

Sciences

$1-1-2015$

\title{
Using hand tracking and electro-tactile stimulation to facilitate learning touch typing
}

Daniel S. Pamungkas

University of Wollongong, dsp572@uowmail.edu.au

Koren Ward

University of Wollongong, koren@uow.edu.au

Follow this and additional works at: https://ro.uow.edu.au/eispapers

Part of the Engineering Commons, and the Science and Technology Studies Commons

Research Online is the open access institutional repository for the University of Wollongong. For further information contact the UOW Library: research-pubs@uow.edu.au 


\title{
Using hand tracking and electro-tactile stimulation to facilitate learning touch typing
}

\author{
Abstract \\ Learning to touch-type can be a more difficult task when the user has acquired incorrect or bad typing \\ practices after many years of ad-hock ke $\backslash$ boarding and computer use. In this paper we introduce a novel \\ training aid to facilitate the learning of touch-typing, particularly the learning of the correct finger-key \\ associations. Our learning system differs to existing touch-typing tutor applications in that we also \\ monitor the finger positions with a hand motion tracking sensor to check that the correct fingers are being \\ used to press the correct keys. Our system also uses electro-neural finger stimulation to enhance learning \\ of the correct finger-key associations. We provide our preliminary experimental results that demonstrate \\ the effectiveness of this approach for learning the correct finger-key associations required for touch- \\ typing

\section{Disciplines} \\ Engineering | Science and Technology Studies

\section{Publication Details} \\ Pamungkas, D. \& Ward, K. (2015). Using hand tracking and electro-tactile stimulation to facilitate learning \\ touch typing. Annual International Conference on Education \& e-Learning (EeL2015) (pp. 95-100).
}




\section{Using Hand Tracking and Electro-tactile Stimulation to Facilitate Learning Touch Typing}

\author{
Daniel S Pamungkas \\ School of Computer Science and Software Engineering \\ University of Wollongong \\ Wollongong, Australia \\ dsp572@uowmail.edu.au
}

\author{
Koren Ward \\ School of Computer Science and Software Engineering \\ University of Wollongong \\ Wollongong, Australia \\ koren@uow.edu.au
}

\begin{abstract}
Learning to touch-type can be a more difficult task when the user has acquired incorrect or bad typing practices after many years of ad-hock keyboarding and computer use. In this paper we introduce a novel training aid to facilitate the learning of touch-typing, particularly the learning of the correct finger-key associations. Our learning system differs to existing touch-typing tutor applications in that we also monitor the finger positions with a hand motion tracking sensor to check that the correct fingers are being used to press the correct keys. Our system also uses electro-neural finger stimulation to enhance learning of the correct finger-key associations. We provide our preliminary experimental results that demonstrate the effectiveness of this approach for learning the correct finger-key associations required for touch-typing.
\end{abstract}

Keywords - touch-typing; associative learning; haptic feedback; electro-tactile feedback; finger motoric learning.

\section{INTRODUCTION}

The proliferation and widespread use of personal computers at home, at work and in education, places considerable demands on students and employees to be able to use the keyboard effectively. Keyboarding involves sequentially pressing the correct keys on the keyboard for entering text, and also performing specialist functions like: "copy", "paste", "page-up", "help", etc. which sometimes involves pressing multiple keys simultaneously.

Touch-typing is considered a subset of keyboarding and involves being able to type accurately on a keyboard with all the fingers while not looking at the keyboard. Touch-typing can be leant either by having traditional classroom style touchtyping lessons or the use of specialized touch-typing tutor applications that enable the user to learn to touch type by performing repetitive typing tasks on the computer.

The advent of the internet and the proliferation of the personal computer has resulted in most people being exposed to the computer and keyboard before they have learnt to touchtype via formal lessons or typing tutor applications. Although, continued general use of the computer and keyboard can help computer users to become familiar with the key positions and be able to enter text without having to look at the keyboard, it rarely results in the correct fingers being used to press the correct keys on the keyboard.

This can result in bad typing habits being acquired like pressing keys with the wrong fingers or using certain fingers too much or too little. Consequently, by the time most students are required to learn to touch-type many of them have acquired bad typing habits which can make correct touch-typing a more frustrating and difficult skill for the student to learn.

To address this problem, we have been experimenting with the learning (or relearning) of proper touch-typing finger-key associations by using hand and finger tracking sensors and electro-tactile feedback. This is achieved by exposing the user to specific keyboard characters via sound and/or visual cues and electrically stimulating the appropriate finger that should be used to action that character on the keyboard. We provide experimental results that show that after repeated exposure to this form of associative learning, the correct finger-character response becomes more reflexive, which can help to reduce the amount of time and effort needed to learn (or relearn) the correct touch-typing finger-key associations.

This paper is organized as follows: Section II provides a brief overview of related work within this field of study. Section III presents the implementation details of our fingercharacter associative learning system. Section IV describes some preliminary experiments we have performed which demonstrate the potential of the proposed associative learning system to assist computer users to lean or improve their touch typing skills. Finally, Section V provides some the concluding remarks and future work to be done.

\section{BACKGROUND}

Learning can be described as the process or act of acquiring knowledge, behaviors or skills and involves stimulus from the environment, reaction and repetition [1]. Much of this process involves the learning of associations that relate one thing to another.

There are two types of associative learning referred to as classical and operant conditioning [2]. Classical conditioning occurs when neutral stimulus is repeatedly presented together with a reflex eliciting stimuli until eventually the neutral 
stimulus elicits a response on its own. Operant conditioning occurs when a certain behavior is either reinforced or punished which results in the behavior becoming habitual or avoided. For example, a hungry dog will naturally do salivation when given food. In a classic experiment, Parlov [3] gave food to a dog which was paired with a tonal sound. After several trials the dog would salivate in response to the tone. This experiment showed that the associative learning process can cause a subject to do something unconsciously or automatically after being given enough reflex eliciting stimuli.

Acquiring motoric skills, such as touch-typing or playing a piano, requires repetitive dexterous finger movements [4]. There are two opposing views as to how children should learn to use the keyboard, namely, the constructivist view and the instructionalist view [5]. The constructivist view emphasises the need for free exploration and discovery. It is based on allowing children to use the keyboard as they wish and aims at focusing children's attention on the cognitive processes. The opposing instructionalist view resembles traditional teaching and regards the constructivist exploration and discovery process as wasteful and likely to induce frustration in the pupils as they "hunt and peck". The fear is that children will learn bad habits that will be difficult to unlearn when they endeavor to learn proper touch typing [6].

Various typing tutor computer applications have become available in recent years. Most have graphical user interfaces that present text on the screen and show the user what keys to press and which fingers to use with the aid of hand and keyboard images. They have various incremental lessons and can also have touch-typing games to keep the user interested and engaged.

Although typing tutor programs can assist users to learn or improve their touch-typing skills they still require considerable repetitive effort from the user, particularly, if the user has acquired incorrect or bad typing habits over a long period of time. Another problem with existing typing tutor programs is that they lack the means for determining if the user is pressing the correct keys with the correct fingers which can allows users to still achieve good scores while maintaining their bad typing habits [2].

Wearable computing devices comprised of gloves fitted with vibration actuators mounted on the fingers have been used to facilitate learning certain motoric skills including playing the piano [7]. As vibro-tactile gloves are somewhat cumbersome and restrict movement, they are mostly used for Passive Haptic Learning (PHL) [8].

Piano PHL is where the user is presented with a sequence of audio musical notes while the appropriate fingers are vibrated in sequence at the same time. When the user thinks he can recall the notes with his fingers, he removes the glove and attempts to play the musical notes on the piano. This PHL piano learning approach has also been tested on patients with spinal cord injuries and shown to have some rehabilitative benefits [7].

Haptic interfaces like vibro-tactile gloves [9], have also been used to train blind people how to type Braille using a custom built Braille keyboard [10]. Braille keyboards have six keys which represent the six dots in Braille alphabetic characters and one key to represent the spacebar. Both hands are used to press the keys and most characters require multiple keys to be pressed simultaneously. Since blind people do not have access to visual cues, haptic finger cues, from vibrotactile gloves provide an ideal substitute. This haptic learning approach also has the benefit that the associations between fingers and characters can be learnt passively, as in PHL, or actively by pressing the keys under the fingers that are vibrated.

These vibro-tactile haptic learning experiments show that haptic stimulus can facilitate learning the finger-key associations needed to play the piano or type in Braille. They also show that these skills can be learnt passively or actively. The disadvantage of using a vibro-tactile glove to stimulate the fingers is that the glove can be cumbersome and can impede dexterous movement of the hands and when fingers near to each other are vibrated simultaneously, it can sometimes be hard to determine if one finger or both fingers are being vibrated. These systems also have no monitoring or tracking of the fingers and therefore are unable to determine if the user is pressing the correct key with the correct finger. The main objective of our research is to overcome these disadvantages and show that passive and active associative learning can also be used to learn touch-typing more effectively.

To improve the learning of touch typing with a computer, we have developed a typing tutor system which is equipped with hand and finger tracking so that the application can check that the correct fingers are being used to press the correct keys. In addition to this, our system is able to deliver electro-tactile stimulus to the fingers to facilitate the passive and active haptic learning of associations between fingers and keys. Our typing tutor has various lessons that expose the user to both passive and active haptic learning for maximum learning effect.

The main components of our haptic associative learning system are a LeapMotion sensor for tracking the hands and fingers [11] and a haptic electro-tactile interface for electrically stimulating the fingers $[12,13,14]$. The LeapMotion sensor is comprised of two IR cameras and 3 IR LEDs and can accurately track the hands and fingers within a 1 meter envelope. The sensor unit is relatively small and can be mounted on the desktop or keyboard to look up at the hands (desktop mode) or above the hands to look down on the hands (HMD mode), as in our system.

Haptic electro-tactile stimulation is based on electrocutaneous stimulation and utilizes electric currents to more directly stimulate nerves in the skin. It can also produce a wide variety of sensations by modulating both the frequency and intensity of the stimulation. As electro-tactile stimulation only requires small electrodes to be placed on the back of the fingers it is less cumbersome than vibro-tactile gloves and does not impede movement of the hands.

In following section, we provide the implementation details of our proposed associative learning typing tutor system. Section IV provides some preliminary experimental results and some concluding remarks are provided in Section V. 


\section{MULTISENSORY LEARNING SYSTEM}

\section{A. Overview}

To test the benefit of adding hand tracking and electrotactile stimulation to a typing tutor application we fitted a PC with a LeapMotion unit (in HMD mode) [11], and a custom built wireless five channel electro-tactile unit for electrically stimulating the fingers, as shown in Figure 1. We also implemented a typing tutor application that utilizes these interfaces and teaches touch-typing via passive and active haptic associative learning. Figure 2 presents a block diagram overview of the proposed touch-typing learning system.

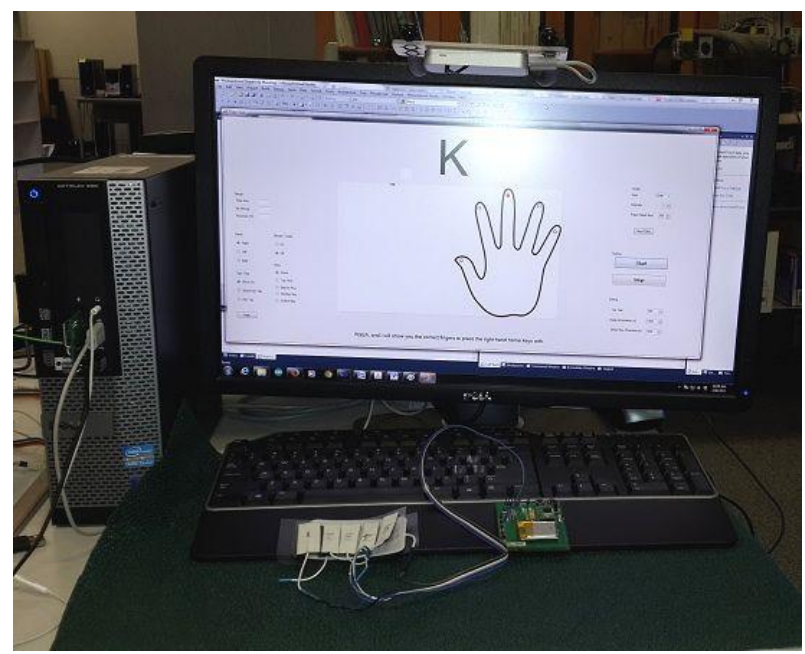

Figure 1. PC with LeapMotion sensor (top) and keyboard and electro-tactile finger stimulation unit (bottom).

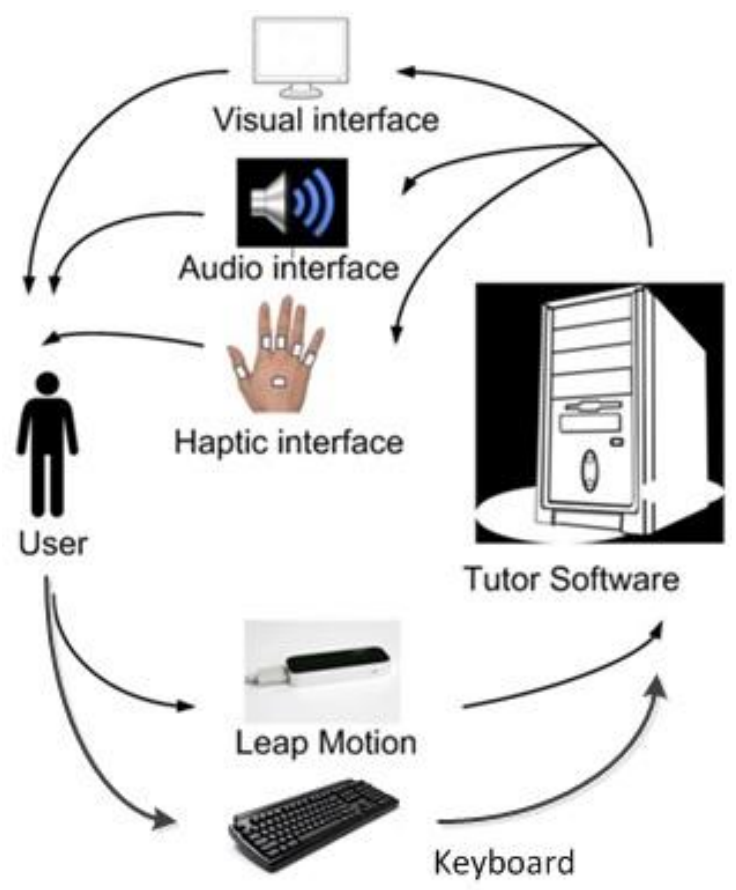

Figure 2. Block diagram of the hand tracking haptic typing tutor system.

\section{B. Handtracking}

To track the movement of the fingers a LeapMotion hand tracking unit is used [11]. This device illuminates the hands with IR LEDs and tracks the hands and fingers in real time using stereo infrared cameras. It has an accuracy of up to 0.1 millimeter in three dimensions [13] and can recognize gestures, like finger "key-taps", "finger swipe", "finger rotate", etc. with low latency. Figure 3 shows the leap motion unit

To enable the hands and fingers to be tracked while typing, we mounted the LeapMotion unit to the monitor, above the hands and keyboard, using a custom built mounting bracket, as shown in Figure 1. Since the LeapMotion unit's cameras are facing down, onto the back of the hands and fingers, the driver software has to be run in HMD mode. To avoid IR reflection from the table and the keyboard, which can interfere with the LeapMotion tracking, we covered the table with dark matting and coated parts of the keyboard with mat black paint, see Figure 1.

\section{Electro-tactile feedback system}

To deliver tactile information from the computer to the user's fingers, two custom-built five channels wireless TENS (Transcutaneous Electrical Nerve Stimulation) system were built, as shown in Figure 4. Each electro-tactile stimulation unit is able to provide five channels of electrical stimulus to the user's fingers with controllable frequency and intensity. The system consists of a USB transmitter, shown in Figure 4a, and a receiver unit shown in Figure $4 \mathrm{~b}$. The transmitter unit transmits finger stimulation data from the computer to the receiver, mounted on the wrists, wirelessly. The receiver unit then converts the finger stimulation data into electrical pulses that are delivered to the fingers via wires and adhesive electrodes placed in the fingers, as shown in Figure 4c. A ground electrode is also placed on the back of the hands as, shown in Figure 5.

This arrangement allows the user to receive up to ten channels of stimulus applied to the fingers concurrently. The stimulus pulses have amplitude of between $40-80 \mathrm{~V}$ and a frequency of $20 \mathrm{~Hz}$, as shown in Figure 6 . The amplitude of the pulses is adjustable via the user interface to suit the user's preference and comfort level. To control the intensity, the pulse width is varied between 10 to $100 \mu \mathrm{s}$. By applying the electrodes to the back of the fingers away from muscles, as shown in Figure 4c, the hands do not experience any contraction from the stimulus. In fact, the stimulus we use is mild and does not affect use of the hands in anyway.

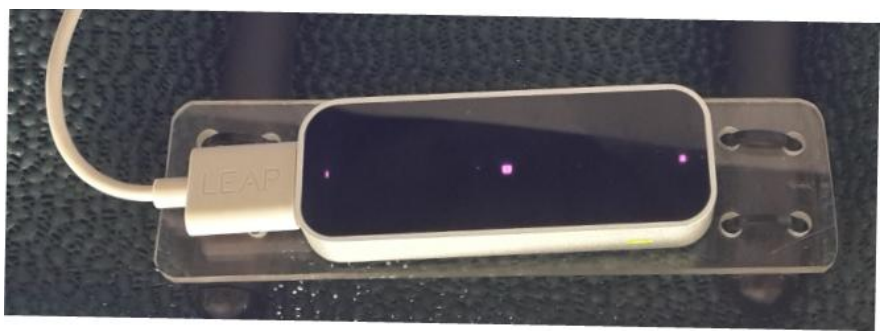

Figure 3. LeapMotion 


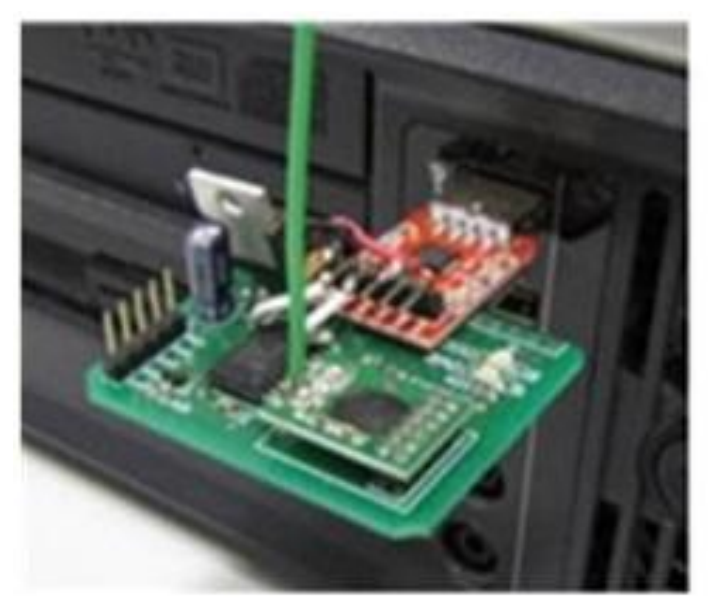

a

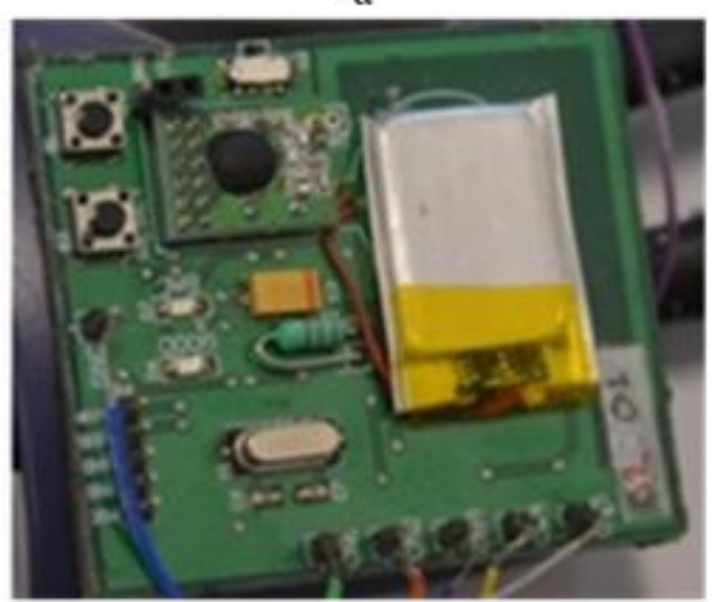

b

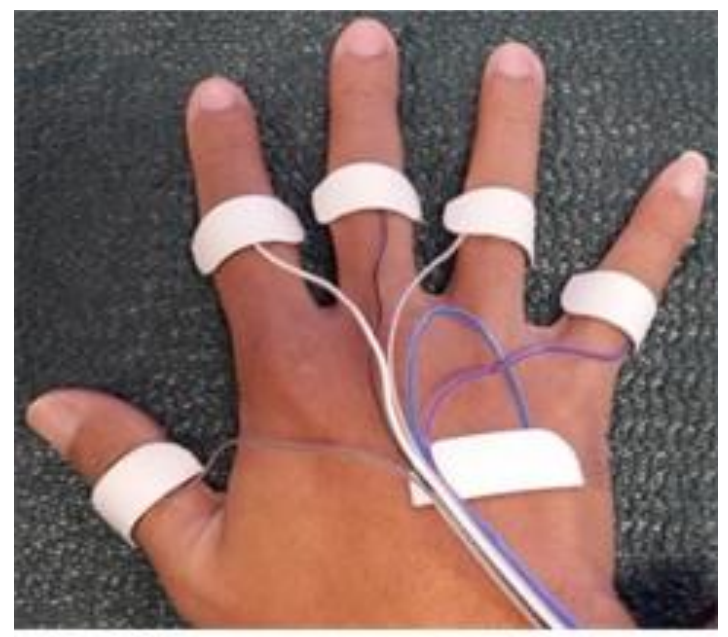

C

Figure 4. Tens System a. Transmiter b. Receiver c. Hand with electrodes

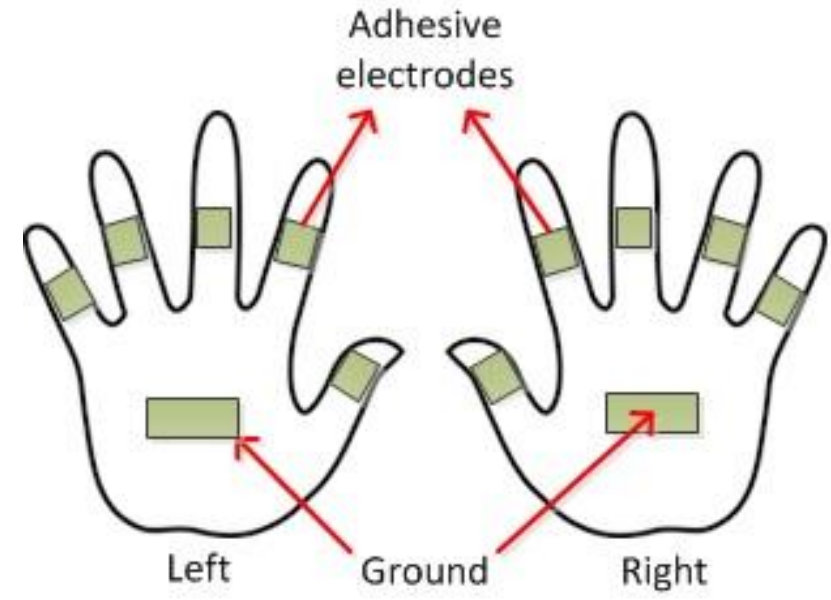

Figure 5. Position of the feedback electrodes

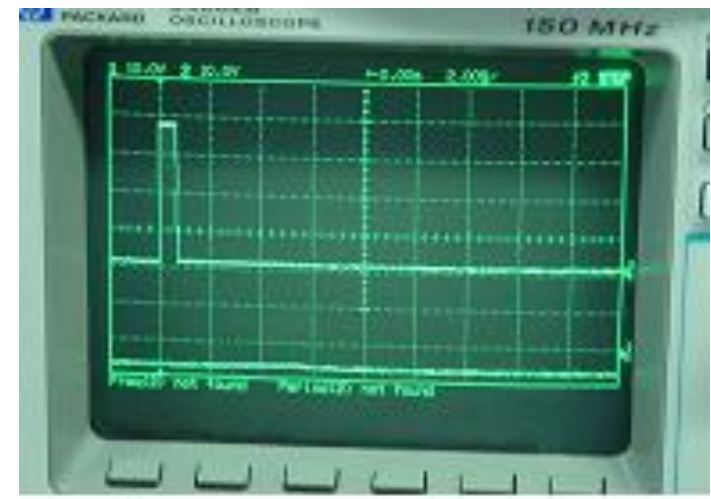

Figure 6. TENS output waveform.

\section{Software}

The software application was designed mainly to teach touch-typing via passive and active haptic learning using electro-tactile stimulation. Tracking the fingers was also done with a LeapMotion unit to check that the correct fingers strike the correct keys and display this information on the screen. Visual and audio cues were also provided to instruct the user on finger-key associations, as in passive and active haptic learning and what to type when striking keys is required.

The user interface of the haptic touch-typing tutor has several controls and options, as shown in Figure 7. Radio buttons are available to allow the user to select which hand(s) to train; namely, left, right or both. If the user selects the right 
hand, then the right hand is depicted on the screen. Likewise, selecting the left hand or both hands depicts images of the left or both hand respectively. There are also radio buttons to select which part of the keyboard to learn. This includes home keys, upper row keys, bottom row keys or number keys.

The user can also select the training method; namely, "passive learning", "active learning with spacebar tapping", or "active learning with key tapping", as explained below. The user can also select to receive the finger cues, via LEDs on the hand image on the screen or via appropriate electro-tactile finger stimulation. Audio of the key characters to press is also available to facilitate teaching the blind to touch-type. Selectable time delays are provided to control the delay between key character cues and the finger cues and the delay between presenting the next character to the user. Controls are also provided for setting the intensity level of the electro-tactile stimulation and testing the electro-tactile stimulation system.

To facilitate the selection of the above mentioned controls, a drop list is provided for selecting the lesson. Each lesson, when selected, will select the appropriate options and delays. "Previous" and "Next" buttons are also provided to move between the lessons.

Lessons are arranged according to which part of the keyboard is learnt, what hand to use and what type of learning to perform. For example, selecting "Home Row - Right Hand Passive Learning" will select the radio buttons and delays appropriate for passive learning with the right hand on the home row keys. The user can then select to have the finger cues provided via electro-tactile stimulation or LEDs on the hand image. Speed and accuracy tests are also provided to determine the user's typing skill both before and after lessons are done. All lessons and tests are timed and scores on accuracy are kept for each lesson and test.

The difference between "passive learning", "active learning with spacebar tapping", or "active learning with key tapping" is explained below.

Passive learning simply involves exposing the user to a key character and indicating the finger to be used to press that key either with LEDs on the hand image or electro-tactile stimulation on the fingers.

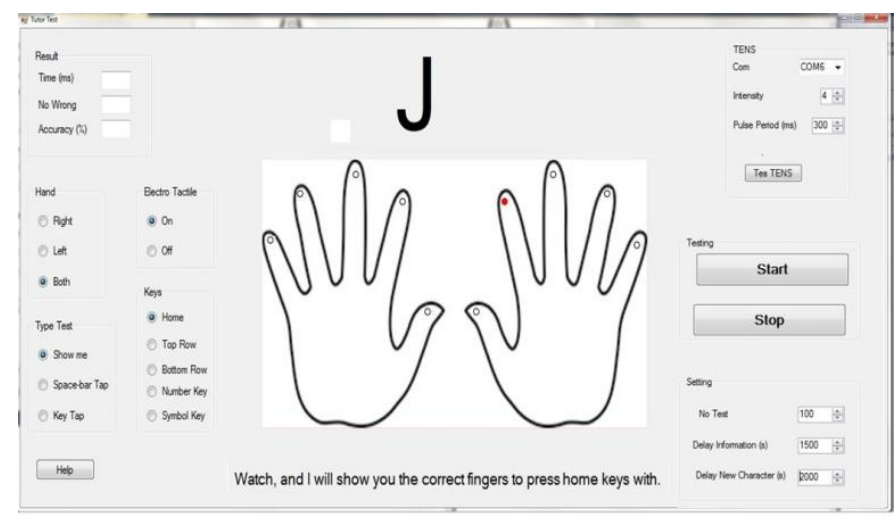

Figure 7. Screenshot of the user interface.
Active learning with spacebar tapping involves exposing the user to a key character, indicating the finger to be used to press that key (either with LEDs on the hand image or electrotactile stimulation on the fingers) and waiting for the user to tap the spacebar with the correct finger. The LeapMotion tracker is used to determine if the correct finger is used to tap the spacebar and the score is adjusted accordingly.

Active learning with key tapping involves exposing the user to a key character, indicating the finger to be used to press that key (either with LEDs on the hand image or electro-tactile stimulation on the fingers) and waiting for the user to tap the appropriate key with the correct finger. The software monitors which key is pressed and the LeapMotion tracker is used to determine if the correct finger is used to tap the key. The score is adjusted accordingly.

\section{EXPERIMENTAL METHOD AND RESUlT}

Our preliminary experiments were aimed primarily at determining the effectiveness of hand tracking for both measuring touch-typing skill levels and determining the effectiveness of haptic (electro-tactile) passive and active learning at improving touch-typing skills.

To do this we conducted a learning trial with twelve university students. All the students claimed that their touchtyping skills had room for improvement. The students were given a preliminary touch-typing speed and accuracy test to determine the level of their touch-typing skills. The students were then divided into two groups (the "test group" and the "control group") which both groups having an equal mix of individuals with varying skill levels.

The test group was given a number of lessons on our typing-tutor application with the electro-tactile stimulation activated. The control group was given exactly the same lessons without electro-tactile stimulation.

Each individual was given instructions on how to use the typing-tutor application followed with one hour of learning with the lessons focused on getting the users to strike the home keys with the correct fingers. All individuals were given speed and accuracy tests both before and after each lesson to gauge the amount of improvement as a result of each lesson.

Analysis of the results revealed that all the subjects, both in the test group and the control group, improved their speed and accuracy at striking the control keys with the correct fingers. On average, the test group achieved an improvement in speed of $20 \%$ and an improvement in accuracy of $50 \%$. The control group on average achieved an improvement in speed of $15 \%$ and accuracy of $20 \%$. In both groups the improvement was more significant on subjects with lower skill levels.

Our results indicate that electro-tactile stimulation can improve both the speed and accuracy of touch-typing lessons. Our experiments also show that hand tracking can facilitate with both the testing and training of touch-typing. 


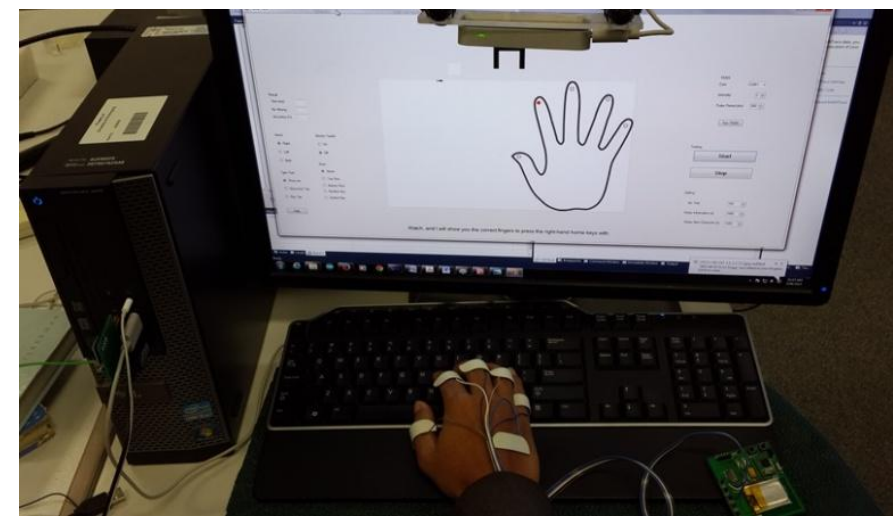

Figure 8. Test subject using our system with electro-tactile stimulation

\section{CONCLUSION}

This paper presents a prototype touch-typing learning system with finger tracking and haptic active and passive learning of finger-key associations. Finger tracking is achieved with a LeapMotion hand tracking unit and custom built finger tracking and finger gesture identification software. Electrotactile stimulation of the fingers is used to achieve haptic passive and active learning of finger-key associations by giving sound and/or visual exposure of keyboard characters and electrically stimulating the appropriate finger that should be used to press the keyboard character.

The experimental results show two benefits from the proposed system. Firstly, repeated exposure to active and passive haptic learning of finger-key associations reduces the amount of time and effort needed to learn touch typing. Secondly, hand and finger tracking hardware and software can facilitate gauging an individual's level of touch-typing skill and can help with the learning of touch-typing by ensuring that the correct fingers are being used to strike the correct keys.

For future work we intent to further evaluate our haptic touch-typing training system on children and adults. We intend also expanding our haptic hand tracking learning method to learning to play musical instruments.

\section{REFERENCES}

[1] Kandel, ER , JH Schwartz and TM Jessell, Principles of Neural Science, New York: McGraw-Hill, 2000.

[2] Bitterman et al. (1983). "Classical Conditioning of Proboscis Extension in Honeybees (Apis mellifera)". J. Comp. Psych 97: 107-119. doi:10.1037/0735-7036.97.2.107.

[3] Pavlov, I,P,. Conditioned reflexes (G.V. Snrep, Trans.). Oxford, Oxford University Press.1927.

[4] P.Fitts and M.Posner, HumanPerformance.Belmont, CA,Brooks/Cole, 1967.

[5] McKinnon, D. H. and Nolan, C. J. P. (1990). Keyboarding, touch typing and computers: What students think. In J. G. Hedberg, J. Steele and M. Mooney (Eds), Converging Technologies: Selected papers from EdTech'90, 59-68. Canberra: AJET Publications.

[6] Hunter, J. H., Begoray J., Benedict G., Bilan B., Jardine G., Rilestone P. \& Weisgerber R. (1988). Writing and word processing: A critical synthesis. Education Technology Unit, Calgary Board of Education.

[7] Markow, T., N. Ramakrishnan, et al. (2010). Mobile Music Touch: Vibration stimulus in hand rehabilitation. Pervasive Computing Technologies for Healthcare (PervasiveHealth), 2010 4th International Conference on.

[8] Kohlsdorf, D.; Starner, T., "Mobile Music Touch: The effect of primary tasks on passively learning piano sequences," Wearable Computers (ISWC), 2010 International Symposium on , vol., no., pp.1,8, 10-13 Oct. 2010

[9] O’Maley, M.K and Gupta. A, Haptic interface, 2008 (pp 25-73) MorganKaufman Publisher.

[10] Seim, C. E., D. Quigley, et al. (2014). Passive haptic learning of typing skills facilitated by wearable computers. CHI '14 Extended Abstracts on Human Factors in Computing Systems. Toronto, Ontario, Canada, ACM: 2203-2208.

[11] Bachmann, D., F. Weichert, and G. Rinkenauer,. "Evaluation of the Leap Motion Controller as a New Contact-Free Pointing Device," Sensors, vol 15, no 1, pp 214-233, 2015.

[12] D. S. Pamungkas and K. Ward, "Tele-operation of A Robot Arm With Electro Tactile Feedback. Advanced Intelligent Mechatronics (AIM), 2013 IEEE/ASME International Conference on, pp. 704-709, Wollongong, 2013.

[13] H. Kajimoto, M Inami, N Kawakami, and S Tachi., "SmartTouch Augmentation of skin Sensation With Electrocutaneous Display. Haptic Interfaces for Virtual Environment and Teleoperator Systems." 2003. HAPTICS 2003 Proceedings. 11th Symposium on, pp. 40-46, March 2003.

[14] M. Peruzzini, M.Germani, and M. Mengoni. "Electro-Tactile Device for Texture Simulation." IEEE/ASME International Conference on Mechatronics and Embedded Systems and Applications (MESA),pp. 178-183, 2012 